\title{
ASYMPTOTIC BEHAVIOR OF SYSTEMS OF LINEAR ORDINARY DIFFERENTIAL EQUATIONS
}

\author{
E. C. TOMASTIK
}

Abstract. Conditions will be placed on the $m \times m$ matrices $G(t)$ and $G_{i}(t)$ to assure that for any integer $k=1, \ldots, n$, the linear differential system

$$
\begin{aligned}
x_{i}^{\prime} & =G_{i}(t) x_{i+1}, \quad i=1, \ldots, n-1, \\
x_{n}^{\prime} & =G(t) x_{1},
\end{aligned}
$$

where the $x_{i}$ are $m \times m$ matrices, has a solution $\left(x_{1}, \ldots, x_{n}\right)$ with the property that $x_{k}(t) \rightarrow I$ (the identity matrix) and if $k<n, x_{i}(t) \rightarrow 0, i=k+1, \ldots, n$, as $t \rightarrow \infty$. Furthermore, important bounds on the $x_{i}(t)$ will be given. Some of these conditions will require that $\int_{a}^{\infty}|G|<\infty$ while others will not. Corollaries will be given for special cases such as $\left(R(t) x^{\prime \prime}\right)^{\prime \prime}=G(t) x$. No selfadjointness conditions are assumed; however, the results are new even in the selfadjoint case.

In this paper the asymptotic behavior of the matrix system

$$
\begin{aligned}
& x_{i}^{\prime}=G_{i}(t) x_{i+1}, \quad i=1,2,3, \\
& x_{4}^{\prime}=G(t) x_{1}
\end{aligned}
$$

will be studied, where $G(t)$ and the $G_{i}(t)$ are $m \times m$ matrices of continuous functions on $[a, \infty), a \geq 0$. For a given integer $k=1,2,3,4$, conditions will be placed on $G(t)$ and the $G_{i}(t)$ which will insure the existence of a matrix solution $\left(x_{1}, x_{2}, x_{3}, x_{4}\right)$ of (1), each $x_{i}$ being an $m \times m$ matrix, such that $x_{k}(t) \rightarrow I$ (the identity matrix) as $t \rightarrow \infty$, and furthermore if $k<4, x_{i}(t) \rightarrow 0$ for $i=k+1, \ldots, 4$, as $t \rightarrow \infty$. Some of these conditions will require that $\int^{\infty}|G|<\infty$, while others will not. Also, important bounds on the $x_{i}(t)$ will be shown to exist.

Extensions are given to

$$
\begin{aligned}
x_{i}^{\prime} & =G_{i}(t) x_{i+1}, \quad i=1, \ldots, n-1, \\
x_{n}^{\prime} & =G(t) x_{1} .
\end{aligned}
$$

The second order scalar case $(m=1, n=2)$, has been studied by Hartman [1], Hartman and Wintner [2], Hille [3] and Wintner [6, 7]. Shreve [5] obtained results for the case $n=2, G_{1}(t)=I, G_{2}(t)$ symmetric, and Rasmussen [4] for the general $n=2$ case.

In case the $G_{i}(t)$ are all nonsingular, (1) can be written as the fourth order equation

$$
\left(R_{3}\left(R_{2}\left(R_{1} x_{1}^{\prime}\right)^{\prime}\right)^{\prime}\right)^{\prime}=G x_{1}
$$

Received by the editors November 12, 1981 and, in revised form, February 22, 1983.

1980 Mathematics Subject Classification. Primary 34C99.

Key words and phrases. Asymptotic behavior, systems of linear differential equations. 
where $R_{i}=G_{i}^{-1}, i=1,2,3$. Two important cases of (2) will be considered. First if $G_{1}=G_{3}=I$ and $R_{2}=R$, then (2) is

$$
\left(R x_{1}^{\prime \prime}\right)^{\prime \prime}=G x_{1}
$$

If $R=I$, then (3) is just

$$
x_{1}^{(\mathrm{iv})}=G(t) x_{1}
$$

Theorem 1. Assume $\int_{a}^{\infty}|G(s)| d s<\infty$ and define $\gamma(t)=\int_{t}^{\infty}|G(s)| d s$. Also assume that

$$
\int_{a}^{\infty}\left|G_{1}(u)\right| \int_{u}^{\infty}\left|G_{2}(v)\right| \int_{v}^{\infty}\left|G_{3}(w)\right| \gamma(w) d w d v d u<\infty .
$$

Then there exists a solution $\left(x_{1}, x_{2}, x_{3}, x_{4}\right)$ of $(1)$ such that $x_{1}(t) \rightarrow I$ and $x_{i}(t) \rightarrow 0$ for $i=2,3,4$. Furthermore there exists $b \geq a$, such that

$$
\begin{gathered}
\left|x_{2}(t)\right| \int_{b}^{t}\left|G_{1}(s)\right| d s \rightarrow 0, \\
\left|x_{3}(t)\right| \int_{b}^{t}\left|G_{2}(u)\right| \int_{b}^{u}\left|G_{1}(v)\right| d v d u \rightarrow 0, \\
\left|x_{4}(t)\right| \int_{b}^{t}\left|G_{3}(u)\right| \int_{b}^{u}\left|G_{2}(v)\right| \int_{b}^{v}\left|G_{1}(w)\right| d w d v d u \rightarrow 0,
\end{gathered}
$$

as $t \rightarrow \infty$.

To establish Theorem 1, write (1) in the integral form

$$
\begin{aligned}
x_{4}(t)= & x_{4}(a)+\int_{a}^{t} G(s) d s x_{1}(b)+\int_{a}^{t} G(s) \int_{b}^{s} G_{1}(u) d u d s x_{2}(c) \\
& +\int_{a}^{t} G(s) \int_{b}^{s} G_{1}(u) \int_{c}^{u} G_{2}(v) d v d u d s x_{3}(d) \\
& +\int_{a}^{t} G(s) \int_{b}^{s} G_{1}(u) \int_{c}^{u} G_{2}(v) \int_{d}^{v} G_{3}(w) x_{4}(w) d w d v d u d s .
\end{aligned}
$$

Since we want $x_{1}(t) \rightarrow I, x_{2}(t) \rightarrow 0, x_{3}(t) \rightarrow 0, x_{4}(t) \rightarrow 0$, we consider the integral equation

(9) $z(t)=-\int_{t}^{\infty} G(s) d s+\int_{t}^{\infty} G(s) \int_{s}^{\infty} G_{1}(u) \int_{u}^{\infty} G_{2}(v) \int_{v}^{\infty} G_{3}(w) z(w) d w d v d u d s$

We must first show that a solution of this equation exists. Toward this end define

$$
\lambda_{1}(t)=\int_{t}^{\infty}\left|G_{1}(s)\right| \int_{s}^{\infty}\left|G_{2}(u)\right| \int_{u}^{\infty}\left|G_{3}(v)\right| \gamma(v) d v d u d s
$$

Set $\lambda=\lambda_{1}(b)$, where $b \geq a$ is large enough that $\lambda_{1}(b)<\frac{1}{2}$. Define successive approximations by $z_{0}(t)=-\int_{t}^{\infty} G(s) d s$,

$$
z_{k+1}(t)=z_{0}(t)+\int_{t}^{\infty} G(s) \int_{s}^{\infty} G_{1}(u) \int_{u}^{\infty} G_{2}(v) \int_{v}^{\infty} G_{3}(w) z_{k}(w) d w d v d u d s .
$$

Of course $\left|z_{0}(t)\right| \leq \gamma(t)$. Now assume that $z_{k}(t)$ is well defined, continuous and satisfies

$$
\left|z_{k}(t)\right| \leq \gamma(t)\left[1+\lambda+\cdots+\lambda^{k}\right], \text { for } t \geq b .
$$


Then

$$
\begin{aligned}
\left|z_{k+1}(t)\right| \leq & \gamma(t)+\int_{t}^{\infty}|G(s)| \int_{b}^{\infty}\left|G_{1}(u)\right| \int_{u}^{\infty}\left|G_{2}(v)\right| \int_{v}^{\infty}\left|G_{3}(w)\right| \gamma(w) d w d v d u d s \\
& \times\left[1+\lambda+\cdots+\lambda^{k}\right]=\gamma(t)\left[1+\lambda+\cdots+\lambda^{k+1}\right] .
\end{aligned}
$$

Thus $z_{k}(t)$ is by induction well defined, continuous, and satisfies (10) for all $k$. Let $\Delta z_{k}(t)=z_{k}(t)-z_{k-1}(t)$ for $k \geq 1$. Then $\left|\Delta z_{1}(t)\right| \leq \gamma(t) \lambda$ for $t \geq b$, and by induction it readily follows that $\left|\Delta z_{k}(t)\right| \leq \gamma(t) \lambda^{k}$, for $t \geq b$. Then $\sum \Delta z_{k}(t)$ converges uniformly on $[b, \infty)$ since $\lambda<\frac{1}{2}$ and $\gamma(t)$ is bounded. Thus $z_{k}(t)$ converges uniformly on $[b, \infty)$ to (say) the continuous function $z(t)$ and $|z(t)| \leq \gamma(t) \sum \lambda^{k}<2 \gamma(t)$. Since the appropriate integrals are bounded, $z(t)$ is a solution to (9). Now define $x_{4}(t)=z(t)$. Then

$$
x_{4}^{\prime}(t)=G(t)\left[I-\int_{t}^{\infty} G_{1}(u) \int_{u}^{\infty} G_{2}(v) \int_{v}^{\infty} G_{3}(w) x_{4}(w) d w d v d u\right] .
$$

Now continue by defining

$$
x_{1}(t)=I-\int_{t}^{\infty} G_{1}(u) \int_{u}^{\infty} G_{2}(v) \int_{v}^{\infty} G_{3}(w) x_{4}(w) d w d v d u
$$

Then certainly since $\left|x_{4}(t)\right|=|z(t)| \leq 2 \gamma(t)$ and $\lambda_{1}(t)<\infty, x_{1}(t) \rightarrow I$ as $t \rightarrow \infty$. Then

$$
x_{1}^{\prime}(t)=G_{1}(t) \int_{t}^{\infty} G_{2}(u) \int_{u}^{\infty} G_{3}(v) x_{4}(v) d v d u
$$

and define $x_{2}(t)=\int_{t}^{\infty} G_{2}(u) \int_{u}^{\infty} G_{3}(v) x_{4}(v) d v d u$. Then

$$
x_{2}^{\prime}(t)=-G_{2}(t) \int_{t}^{\infty} G_{3}(s) x_{4}(s) d s
$$

and define $x_{3}(t)=-\int_{t}^{\infty} G_{3}(s) x_{4}(s) d s$. Since $x_{3}^{\prime}(t)=G_{3}(t) x_{4}(t),\left(x_{1}, x_{2}, x_{3}, x_{4}\right)$ is a solution to (1) with $x_{1}(t) \rightarrow I, x_{2}(t) \rightarrow 0, x_{3}(t) \rightarrow 0, x_{4}(t) \rightarrow 0$.

It now remains to establish the bounds given in the theorem for $x_{2}, x_{3}$ and $x_{4}$. Toward this end, make the following definitions:

$$
\lambda_{3}(t)=\int_{t}^{\infty}\left|G_{3}(s)\right| \gamma(s) d s, \quad \lambda_{2}(t)=\int_{t}^{\infty}\left|G_{2}(s)\right| \lambda_{3}(s) d s .
$$

Notice that $\lambda_{1}(t)=\int_{t}^{\infty}\left|G_{1}(s)\right| \lambda_{2}(s) d s$. We shall now make use of the fact that if $f(t)$ and $g(t)$ are nonnegative continuous functions and $g(t)$ is nonincreasing, $g(t) \rightarrow 0$ as $t \rightarrow \infty$, and $\int_{b}^{\infty} f(s) g(s) d s<\infty$, then $g(t) \int_{b}^{t} f(s) d s \rightarrow 0$ as $t \rightarrow \infty$ (cf. Rasmussen [4, p. 14]). It follows that since $\lambda_{1}(b)<\infty, \lambda_{2}(t)$ is nonincreasing and $\lambda_{2}(t) \rightarrow 0$ as $t \rightarrow \infty$, then $\lambda_{2}(t) \int_{b}^{t}\left|G_{1}(s)\right| d s \rightarrow 0$ as $t \rightarrow \infty$. But, by definition of $x_{2}(t)$,

$$
\begin{aligned}
\left|x_{2}(t)\right| & \leq \int_{t}^{\infty}\left|G_{2}(u)\right| \int_{u}^{\infty}\left|G_{3}(v)\right|\left|x_{4}(v)\right| d v d u \\
& \leq 2 \int_{t}^{\infty}\left|G_{2}(u)\right| \int_{u}^{\infty}\left|G_{3}(v)\right| \gamma(v) d v d u=2 \lambda_{2}(t) .
\end{aligned}
$$

Thus

$$
\left|x_{2}(x)\right| \int_{b}^{t}\left|G_{1}(s)\right| d s \leq 2 \lambda_{2}(t) \int_{b}^{t}\left|G_{1}(s)\right| d s \rightarrow 0 \text { as } t \rightarrow \infty .
$$

This establishes the first limit in Theorem 1, i.e., (5). 
Now by integrating by parts,

$$
\begin{array}{r}
\int_{b}^{M}\left|G_{1}(s)\right| \lambda_{2}(s) d s=\lambda_{2}(M) \int_{b}^{M}\left|G_{1}(s)\right| d s-\int_{b}^{M} \lambda_{2}^{\prime}(s) \int_{b}^{s}\left|G_{1}(u)\right| d u d s \\
=\lambda_{2}(M) \int_{b}^{M}\left|G_{1}(s)\right| d s+\int_{b}^{M}\left|G_{2}(s)\right| \lambda_{3}(s) \int_{b}^{s}\left|G_{1}(u)\right| d u d s .
\end{array}
$$

Since the first term on the right-hand side of (11) has already been shown to go to zero as $M$ goes to infinity, we have

$$
\int_{b}^{\infty}\left|G_{2}(s)\right| \int_{b}^{s}\left|G_{1}(u)\right| d u \lambda_{3}(s) d s=\int_{b}^{\infty}\left|G_{1}(s)\right| \lambda_{2}(s) d s=\lambda_{1}(b)<\infty .
$$

Since $\lambda_{3}(t)$ is nonincreasing and $\lambda_{3}(t) \rightarrow 0$ as $t \rightarrow \infty$, it follows that

$$
\lambda_{3}(t) \int_{b}^{t}\left|G_{2}(s)\right| \int_{b}^{s}\left|G_{1}(u)\right| d u d s \rightarrow 0 \text { as } t \rightarrow \infty .
$$

By definition of $x_{3}(t),\left|x_{3}(t)\right| \leq 2 \int_{t}^{\infty}\left|G_{3}(s)\right| \gamma(s) d s=2 \lambda_{3}(t)$. Thus if $t \geq b$, then

$$
\begin{aligned}
& \left|x_{3}(t)\right| \int_{b}^{t}\left|G_{2}(s)\right| \int_{b}^{s}\left|G_{1}(u)\right| d u d s \\
& \quad \leq 2 \lambda_{3}(t) \int_{b}^{t}\left|G_{2}(s)\right| \int_{b}^{s}\left|G_{1}(u)\right| d u d s \rightarrow 0 \text { as } t \rightarrow \infty
\end{aligned}
$$

This establishes (6) in Theorem 1.

Integrating the second term in (11) by parts yields

$$
\begin{aligned}
\int_{b}^{M}\left|G_{1}(s)\right| \lambda_{2}(s) d s= & \lambda_{2}(M) \int_{b}^{M}\left|G_{1}(s)\right| d s \\
& +\lambda_{3}(M) \int_{b}^{M}\left|G_{2}(s)\right| \int_{b}^{s}\left|G_{1}(u)\right| d u d s \\
& +\int_{b}^{M}\left|G_{3}(s)\right| \gamma(s) \int_{b}^{s}\left|G_{2}(u)\right| \int_{b}^{u}\left|G_{1}(v)\right| d v d u d s .
\end{aligned}
$$

Since we have already shown that the first two terms on the right-hand side of this last equation go to zero as $M$ goes to infinity and since $\lambda_{1}(b)<\infty$, we obtain

$$
\int_{b}^{\infty}\left|G_{3}(s)\right| \int_{b}^{s}\left|G_{2}(u)\right| \int_{b}^{u}\left|G_{1}(v)\right| d v d u \gamma(s) d s<\infty .
$$

But since $\gamma(t)$ is nonincreasing and $\gamma(t) \rightarrow 0$ as $t \rightarrow \infty$,

$$
\gamma(t) \int_{b}^{t}\left|G_{3}(s)\right| \int_{b}^{s}\left|G_{2}(u)\right| \int_{b}^{u}\left|G_{1}(v)\right| d v d u d s \rightarrow 0 \quad \text { as } t \rightarrow \infty .
$$

But since $\left|x_{4}(t)\right| \leq 2 \gamma(t)$, this last limit then readily implies (7). This completes the proof of Theorem 1 .

The following corollaries are obtained by applying Theorem 1 to equations (4) and (3), respectively.

Corollary 1. If $\int_{a}^{\infty} \int_{u}^{\infty} \int_{v}^{\infty} \int_{w}^{\infty}|G(s)| d s d w d v d u<\infty$, then there exists a solution $x(t)$ of the matrix equation $x^{(\mathrm{iv})}=G(t) x$, such that $x(t) \rightarrow I, t\left|x^{\prime}(t)\right| \rightarrow 0$, $t^{2}\left|x^{\prime \prime}(t)\right| \rightarrow 0, t^{3}\left|x^{\prime \prime \prime}(t)\right| \rightarrow 0$, as $t \rightarrow \infty$. 
Corollary 2. If $\int_{a}^{\infty} \int_{u}^{\infty}\left|R^{-1}(v)\right| \int_{v}^{\infty} \int_{w}^{\infty}|G(s)| d s d w d v d u<\infty$, then there exists $b \geq a$ and a solution $x$ of the matrix equation $\left(R(t) x^{\prime \prime}\right)^{\prime \prime}=G(t) x$, such that $x(t) \rightarrow$ $I, t\left|x^{\prime}(t)\right| \rightarrow 0$,

$$
\left|R(t) x^{\prime \prime}(t)\right| \int_{b}^{t} s\left|R^{-1}(s)\right| d s \rightarrow 0, \quad\left|\left(R(t) x^{\prime \prime}(t)\right)^{\prime}\right| \int_{b}^{t} \int_{b}^{u} s\left|R^{-1}(s)\right| d s d u \rightarrow 0,
$$

as $t \rightarrow \infty$.

To establish the next to the last limit in Corollary 2, notice that Theorem 1 implies that

$$
\left|R(t) x^{\prime \prime}(t)\right| \int_{b}^{t}(s-b)\left|R^{-1}(s)\right| d s \rightarrow 0 \quad \text { as } t \rightarrow \infty
$$

and also $\left|R(t) x^{\prime \prime}(t)\right| \rightarrow 0$ as $\rightarrow \infty$. But

$$
\begin{aligned}
& \left|R(t) x^{\prime \prime}(t)\right| \int_{b}^{t}(s-b)\left|R^{-1}(s)\right| d s \\
& \quad=\left|R(t) x^{\prime \prime}(t)\right|\left[\int_{b}^{2 b}(s-b)\left|R^{-1}(s)\right| d s+\int_{2 b}^{t}(s-b)\left|R^{-1}(s)\right| d s\right]
\end{aligned}
$$

and hence $\left|R(t) x^{\prime \prime}(t)\right| \int_{2 b}^{t}(s-b)\left|R^{-1}(s)\right| d s \rightarrow 0$ as $t \rightarrow \infty$. But

$$
\left|R(t) x^{\prime \prime}(t)\right| \int_{2 b}^{t} b\left|R^{-1}(s)\right| d s \leq\left|R(t) x^{\prime \prime}(t)\right| \int_{2 b}^{t}(s-b)\left|R^{-1}(s)\right| d s \rightarrow 0
$$

as $t \rightarrow \infty$ and it follows that $\left|R(t) x^{\prime \prime}(t)\right| \int_{b}^{t} b\left|R^{-1}(s)\right| d s \rightarrow 0$ as $t \rightarrow \infty$. This together with $\left|R(t) x^{\prime \prime}(t)\right| \int_{b}^{t}(s-b)\left|R^{-1}(s)\right| d s \rightarrow 0$ as $t \rightarrow \infty$ then establishes the next to the last limit in Corollary 2. The last limit follows in a similar manner.

Theorem 2. Assume that $\int_{a}^{\infty}|G(s)| \int_{a}^{s}\left|G_{3}(u)\right| d u d s<\infty$ and

$$
\int_{a}^{\infty}\left|G_{2}(s)\right| \int_{s}^{\infty}\left|G_{3}(u)\right| \gamma(u) d u d s<\infty
$$

where $\gamma(t)=\int_{t}^{\infty}|G(s)| \int_{a}^{s}\left|G_{1}(u)\right| d u d s$. Given any $\delta>1$ there exists $a b \geq a$ and $a$ solution $\left(x_{1}, x_{2}, x_{3}, x_{4}\right)$ of $(1)$ such $x_{2}(t) \rightarrow I, x_{3}(t) \rightarrow 0, x_{4}(t) \rightarrow 0$, as $t \rightarrow \infty$ and

$$
\begin{gathered}
\left|x_{1}(t)\right| \leq \delta \int_{b}^{t}\left|G_{1}(s)\right| d s, \\
\left|x_{3}(t)\right| \int_{b}^{t}\left|G_{2}(s)\right| d s \rightarrow 0 \text { as } t \rightarrow \infty, \\
\left|x_{4}(t)\right| \int_{b}^{t}\left|G_{3}(s)\right| \int_{b}^{s}\left|G_{2}(u)\right| d u d s \rightarrow 0 \text { as } t \rightarrow \infty .
\end{gathered}
$$

Recalling (8) and making note that we want $x_{2} \rightarrow I, x_{3} \rightarrow 0, x_{4} \rightarrow 0$, we consider the integral equation

$$
\begin{aligned}
z(t)= & -\int_{t}^{\infty} G(s) \int_{b}^{s} G_{1}(u) d u d s \\
& -\int_{t}^{\infty} G(s) \int_{b}^{s} G_{1}(u) \int_{u}^{\infty} G_{2}(v) \int_{v}^{\infty} G_{3}(w) z(w) d w d v d u d s
\end{aligned}
$$


For any $b \geq a$, define $\lambda_{2}(b)$ to be $\int_{b}^{\infty}\left|G_{2}(s)\right| \int_{s}^{\infty}\left|G_{3}(u)\right| \gamma_{b}(u) d u d s$, where

$$
\gamma_{b}(t)=\int_{t}^{\infty}|G(s)| \int_{b}^{s}\left|G_{1}(u)\right| d u d s
$$

Take $b$ so large that $\lambda=\lambda_{2}(b)$ satisfies $\lambda<1$ and $1 /(1-\lambda)<\delta$ (or $1+\delta \lambda<\delta$ ). Define successive approximations for (12) to be

$$
\begin{gathered}
z_{0}(t)=-\int_{t}^{\infty} G(s) \int_{b}^{s} G_{1}(u) d u d s \\
z_{k+1}(t)=z_{0}(t)-\int_{t}^{\infty} G(s) \int_{b}^{s} G_{1}(u) \int_{u}^{\infty} G_{2}(v) \int_{v}^{\infty} G_{3}(w) z_{k}(w) d w d v d u d s
\end{gathered}
$$

The proof now proceeds precisely as in the proof of Theorem 1 . We obtain a solution to (12) and $|z(t)| \leq \gamma_{b}(t) \sum \lambda^{i}=\gamma_{b}(t) /(1-\lambda)<\delta \gamma_{b}(t)$. Define $x_{4}(t)=z(t)$ and make the natural definitions (as in Theorem 1)

$$
\begin{gathered}
x_{1}(t)=\int_{b}^{t} G_{1}(s) d s+\int_{b}^{t} G_{1}(s) \int_{s}^{\infty} G_{2}(u) \int_{u}^{\infty} G_{3}(v) x_{4}(v) d v d u d s \\
x_{2}(t)=I+\int_{t}^{\infty} G_{2}(s) \int_{s}^{\infty} G_{3}(u) x_{4}(u) d u d s \\
x_{3}(t)=-\int_{t}^{\infty} G_{3}(s) x_{4}(s) d s .
\end{gathered}
$$

We notice that $\left|x_{1}(t)\right| \leq(1+\delta \lambda) \int_{b}^{t}\left|G_{1}(s)\right| d s \leq \delta \int_{b}^{t}\left|G_{1}(s)\right| d s$, which is the bound on $\left|x_{1}(t)\right|$ in Theorem 2 .

Now define $\lambda_{3}(t)=\int_{t}^{\infty}\left|G_{3}(s)\right| \gamma_{b}(s) d s$ which is finite by hypothesis. Then also by hypothesis, $\lambda_{2}(t)=\int_{t}^{\infty}\left|G_{2}(s)\right| \lambda_{3}(s) d s<\infty$. But since $\lambda_{3}(t)$ is nonincreasing and $\lambda_{3}(t) \rightarrow 0$ as $t \rightarrow \infty$, then $\lambda_{3}(t) \int_{b}^{t}\left|G_{2}(s)\right| d s \rightarrow 0$ as $t \rightarrow \infty$. By definition of $x_{3}(t)$ and since $\left|x_{4}(t)\right| \leq \delta \gamma_{b}(t),\left|x_{3}(t)\right| \leq \delta \lambda_{3}(t)$. Thus

$$
\left|x_{3}(t)\right| \int_{b}^{t}\left|G_{2}(s)\right| d s \leq \delta \lambda_{3}(t) \int_{b}^{t}\left|G_{2}(s)\right| d s \rightarrow 0 \text { as } t \rightarrow \infty,
$$

which establishes the limit in Theorem 2 that involves $x_{3}$.

To establish the last limit in Theorem 2, integrate the following term by parts and obtain

$$
\int_{b}^{M}\left|G_{2}(s)\right| \lambda_{3}(s) d s=\lambda_{3}(M) \int_{b}^{M}\left|G_{2}(s)\right| d s+\int_{b}^{M}\left|G_{3}(s)\right| \gamma_{b}(s) \int_{b}^{s}\left|G_{2}(u)\right| d u d s .
$$

Recall that we have already shown that the first term on the right-hand side of the last equation goes to zero as $M \rightarrow \infty$; thus

$$
\lambda_{2}(b)=\int_{b}^{\infty}\left|G_{2}(s)\right| \lambda_{3}(s) d s=\int_{b}^{\infty}\left|G_{3}(s)\right| \int_{b}^{s}\left|G_{2}(u)\right| d u \gamma_{b}(s) d s .
$$

Since $\gamma_{b}(t)$ is nonincreasing and $\gamma_{b}(t) \rightarrow 0$ as $t \rightarrow \infty$, it follows that

$$
\gamma_{b}(t) \int_{b}^{t}\left|G_{3}(s)\right| \int_{b}^{s}\left|G_{2}(u)\right| d u d s \rightarrow 0 \text { as } t \rightarrow \infty .
$$

Then

$$
\left|x_{4}(t)\right| \int_{b}^{t}\left|G_{3}(s)\right| \int_{b}^{s}\left|G_{2}(u)\right| d u d s \leq \delta \gamma_{b}(t) \int_{b}^{t}\left|G_{3}(s)\right| \int_{b}^{s}\left|G_{2}(u)\right| d u d s \rightarrow 0
$$

as $t \rightarrow \infty$. This establishes the last limit in Theorem 2 and completes the proof. 
Corollary. If $\int_{a}^{\infty} \int_{u}^{\infty} \int_{v}^{\infty} s|G(s)| d s d v d u<\infty$ and $\delta>1$, then there exists a solution of $x^{(\mathrm{iv})}=G(t) x$ such that $|x(t)| \leq \delta t$ and $x^{\prime}(t) \rightarrow I, t\left|x^{\prime \prime}(t)\right| \rightarrow 0, t^{2}\left|x^{\prime \prime \prime}(t)\right| \rightarrow$ 0 as $t \rightarrow \infty$.

Theorem 3. Assume that $\int_{a}^{\infty}|G(s)| \int_{a}^{s}\left|G_{1}(u)\right| \int_{a}^{u}\left|G_{2}(v)\right| d v d u d s<\infty$ and

$$
\int_{a}^{\infty}\left|G_{3}(s)\right| \gamma(s) d s<\infty
$$

where $\gamma(t)=\int_{t}^{\infty}|G(s)| \int_{a}^{s}\left|G_{1}(s)\right| \int_{a}^{u}\left|G_{2}(v)\right| d v d u d s$. Given any $\delta>1$ there exists a $b \geq a$ and $a$ solution $\left(x_{1}, x_{2}, x_{3}, x_{4}\right)$ of (1) such that $x_{3}(t) \rightarrow I$ and $x_{4}(t) \rightarrow 0$ as $t \rightarrow \infty$ and

$$
\begin{gathered}
\left|x_{1}(t)\right| \leq \delta \int_{b}^{t}\left|G_{1}(s)\right| \int_{b}^{s}\left|G_{2}(u)\right| d u d s, \\
\left|x_{2}(t)\right| \leq \delta \int_{b}^{t}\left|G_{2}(s)\right| d s \\
\left|x_{4}(t)\right| \int_{b}^{t}\left|G_{3}(s)\right| d s \rightarrow 0 \text { as } t \rightarrow \infty .
\end{gathered}
$$

To prove Theorem 3 one considers the integral equation

$$
\begin{aligned}
z(t)= & -\int_{t}^{\infty} G(s) \int_{b}^{s} G_{1}(u) \int_{b}^{u} G_{2}(v) d v d u d s \\
& +\int_{t}^{\infty} G(s) \int_{b}^{s} G_{1}(u) \int_{v}^{u} G_{2}(v) \int_{v}^{\infty} G_{3}(w) z(w) d w d v d u d s
\end{aligned}
$$

The proof proceeds just as in Theorems 1 and 2 .

Corollary. If $\int_{a}^{\infty} \int_{u}^{\infty} s^{2}|G(s)| d s d u<\infty$ and $\delta>1$, then there exists a solution of $x^{(\mathrm{iv})}=G(t) x$ such that $|x(t)| \leq \frac{1}{2} \delta t^{2},\left|x^{\prime}(t)\right| \leq \delta t, x^{\prime \prime}(t) \rightarrow I, t\left|x^{\prime \prime \prime}(t)\right| \rightarrow 0$ as $t \rightarrow \infty$.

THEOREM 4. If $\int_{a}^{\infty}|G(s)| \int_{a}^{s}\left|G_{1}(u)\right| \int_{a}^{u}\left|G_{2}(v)\right| \int_{a}^{v}\left|G_{3}(w)\right| d w d v d u d s<\infty$ and $\delta>1$, then there exists a solution $\left(x_{1}, x_{2}, x_{3}, x_{4}\right)$ of $(1)$ such that $x_{4}(t) \rightarrow I$ as $t \rightarrow \infty$ and

$$
\begin{gathered}
\left|x_{1}(t)\right| \leq \delta \int_{b}^{t}\left|G_{1}(s)\right| \int_{b}^{s}\left|G_{2}(u)\right| \int_{b}^{u} G_{3}(v) \mid d v d u d s \\
\left|x_{2}(t)\right| \leq \delta \int_{b}^{t}\left|G_{2}(s)\right| \int_{b}^{s}\left|G_{3}(u)\right| d u d s \\
\left|x_{3}(t)\right| \leq \delta \int_{b}^{t}\left|G_{3}(s)\right| d s .
\end{gathered}
$$

To prove Theorem 4, one considers

$$
z(t)=I+\int_{b}^{t} G(s) \int_{b}^{s} G_{1}(u) \int_{b}^{u} G_{2}(v) \int_{b}^{v} G_{3}(w) z(w) d w d v d u d s
$$

and the successive approximations given by $z_{0}(t)=I$,

$$
z_{k+1}(t)=I+\int_{b}^{t} G(s) \int_{b}^{s} G_{1}(u) \int_{b}^{u} G_{2}(v) \int_{b}^{v} G_{3}(w) z_{k}(w) d w d v d u d s .
$$

Define $\lambda(b)$ to be $\int_{b}^{\infty}|G(s)| \int_{b}^{s}\left|G_{1}(u)\right| \int_{b}^{u}\left|G_{2}(v)\right| \int_{b}^{v}\left|G_{3}(w)\right| d w d v d u d s$ and pick $b$ so large that $\lambda=\lambda(b)$ is so small that $\lambda<1,1 /(1-\lambda) \leq \sqrt{\delta}$ and if $A$ is any $m \times m$ 
matrix with $|A-I| \leq \lambda$ then $\operatorname{det} A \neq 0$ and $\left|A^{-1}\right| \leq \sqrt{\delta}$. Actually it is easy to see using well-known theorems on lower bounds of $|\operatorname{det} A|$ of matrices $A$ dominated by diagonal elements that if $|A-I|<1 / m$, then $|\operatorname{det} A| \geq(1 / m)^{m}>0$. (Use the matrix norm $|A|=\left|\left(A_{i j}\right)\right|=\max _{i} \sum_{j}\left|A_{i j}\right|$.) That we can make $\left|A^{-1}\right| \leq \sqrt{\delta}$ follows simply from the continuity of the inverse operation and of the norm operation. Proceeding as before, one readily obtains a solution to (13) with $|z(t)| \leq|I| \sum \lambda^{i}=$ $1 /(1-\lambda)<\sqrt{\delta}$. We must show that $\lim z(t)$ exists. Toward this end consider any $t_{2}>t_{1}$, then

$$
\begin{aligned}
\left|z\left(t_{2}\right)-z\left(t_{1}\right)\right| & \leq \sqrt{\delta} \int_{t_{1}}^{t_{2}}|G(s)| \int_{b}^{s}\left|G_{1}(u)\right| \int_{b}^{u}\left|G_{2}(v)\right| \int_{b}^{v}\left|G_{3}(w)\right| d w d v d u d s \\
& \leq \sqrt{\delta} \lambda\left(t_{1}\right) \rightarrow 0 \text { as } t_{1} \rightarrow \infty
\end{aligned}
$$

This shows that $\lim z(t)=L$ exists. But since $|z(t)-I| \leq \lambda,|L-I| \leq \lambda$ and by choice of $\lambda$, det $L \neq 0$ and $\left|L^{-1}\right| \leq \sqrt{\delta}$. Now define $x_{4}(t)=z(t) L^{-1}$ so that $x_{4}(t) \rightarrow I$ as $t \rightarrow \infty$. Also $\left|x_{4}(t)\right| \leq|z(t)|\left|L^{-1}\right| \leq \delta$. Define

$$
x_{1}(t)=\int_{b}^{t} G_{1}(u) \int_{b}^{u} G_{2}(v) \int_{b}^{v} G_{3}(w) x_{4}(w) d w d v d u
$$

and it follows immediately that

$$
\left|x_{1}(t)\right| \leq \delta \int_{b}^{t}\left|G_{1}(u)\right| \int_{b}^{u}\left|G_{2}(v)\right| \int_{b}^{v}\left|G_{3}(w)\right| d w d v d u .
$$

The other inequalities in Theorem 4 follow in a similar manner.

Corollary. If $\int_{a}^{\infty} s^{3}|G(s)| d s<\infty$ and $\delta>1$, there exists a solution of $x^{(\mathrm{iv})}=$ $G(t) x$ such that $|x(t)| \leq \frac{1}{6} \delta t^{3},\left|x^{\prime}(t)\right| \leq \frac{1}{2} \delta t^{2},\left|x^{\prime \prime}(t)\right| \leq \delta t, x^{\prime \prime \prime}(t) \rightarrow I$ as $t \rightarrow \infty$.

By certain simple rearrangements of the equations in (1), twelve more asymptotic results can be obtained. For example, if the last equation in (1) is made the first equation, we obtain

$$
x_{4}^{\prime}=G x_{1}, \quad x_{1}^{\prime}=G_{1} x_{2}, \quad x_{2}^{\prime}=G_{2} x_{3}, \quad x_{3}^{\prime}=G_{3} x_{4} .
$$

Similarly, the other two rearrangements are

$$
x_{3}^{\prime}=G_{3} x_{4}, \quad x_{4}^{\prime}=G x_{1}, \quad x_{1}^{\prime}=G_{1} x_{2}, \quad x_{2}^{\prime}=G_{2} x_{3},
$$

and

$$
x_{2}^{\prime}=G_{2} x_{3}, \quad x_{3}^{\prime}=G_{3} x_{4}, \quad x_{4}^{\prime}=G x_{1}, \quad x_{1}^{\prime}=G_{1} x_{2} .
$$

Each of the four theorems already given can now be applied to each of the three previous equations to obtain an additional twelve asymptotic results. As an example of how this works consider (15). In equation (15), define $z_{1}=x_{3}, z_{2}=x_{4}, z_{3}=$ $x_{1}, z_{4}=x_{2}, H_{1}=G_{3}, H_{2}=G, H_{3}=G_{1}, H=G_{2}$. Then (15) is just

$$
z_{1}^{\prime}=H_{1} z_{2}, \quad z_{2}^{\prime}=H_{2} z_{3}, \quad z_{3}^{\prime}=H_{3} z_{4}, \quad z_{4}^{\prime}=H z_{1}
$$

which is in exactly the form of (1). Each of the previous four theorems can now be applied to (17) to obtain different asymptotic results for (1). Let us further illustrate this by applying Theorem 3 to (17). 
THEOREM 5. If

$$
\int_{a}^{\infty}\left|G_{1}(s)\right| \int_{s}^{\infty}\left|G_{2}(u)\right| \int_{a}^{u}\left|G_{3}(v)\right| \int_{a}^{v}|G(w)| d w d v d u d s<\infty
$$

and $\delta>1$, then there exists $a b \geq a$ and a solution $\left(x_{1}, x_{2}, x_{3}, x_{4}\right)$ of (1) such that $x_{1}(t) \rightarrow I$ and

$$
\begin{gathered}
\left|x_{2}(t)\right| \int_{b}^{t}\left|G_{1}(s)\right| d s \rightarrow 0 \text { as } t \rightarrow \infty \\
\left|x_{3}(t)\right| \leq \delta \int_{b}^{t}\left|G_{3}(s)\right| \int_{b}^{t}|G(u)| d u d s, \\
\left|x_{4}(t)\right| \leq \delta \int_{b}^{t}|G(s)| d s .
\end{gathered}
$$

Corollary. If $\int_{a}^{\infty} \int_{s}^{\infty}\left|R^{-1}(u)\right| \int_{a}^{u} \int_{a}^{v}|G(w)| d w d v d u<\infty$ and $\delta>1$, then there exists $b \geq a$ and a solution $x$ of $\left(R x^{\prime \prime}\right)^{\prime \prime}=G x$ such that $x(t) \rightarrow I, t\left|x^{\prime}(t)\right| \rightarrow 0$ as $t \rightarrow \infty$ and

$$
\left|R(t) x^{\prime \prime}(t)\right| \leq \delta \int_{b}^{t} \int_{b}^{s}|G(u)| d u d s, \quad\left|\left(R(t) x^{\prime \prime}(t)\right)^{\prime}\right| \leq \delta \int_{b}^{t}|G(s)| d s .
$$

The advantage of a result such as Theorem 5 (or the Corollary to Theorem 5) is that it does not require that $\int^{\infty}|G|<\infty$; however, it does require that $\int^{\infty}\left|G_{2}\right|<\infty$ (or $\int^{\infty}\left|R^{-1}\right|<\infty$ ). The advantage of Theorem 3 (or the Corollary to Theorem 3) is that it does not require that $\int^{\infty}\left|G_{2}\right|<\infty$ (or $\int^{\infty} \mid R^{-1}<\infty$ ), but it does require that $\int^{\infty}|G|<\infty$. In the same manner the advantage of the other eleven results alluded to above, also do not require that $\int^{\infty}|G|<\infty$.

The rearrangement procedure given here is equivalent to replacing the integral equation (8), which involves $x_{4}$, with an equation involving only one of the terms $x_{1}, x_{2}$ or $x_{3}$.

The previous results given for $n=4$ can readily be extended to any $n$. For example, Theorem 1 can be generalized as that

Theorem A. Assume $\int_{a}^{\infty}|G(s)|<\infty$ and define $\gamma(t)=\int_{t}^{\infty}|G(s)| d s$. Also assume

$$
\int_{a}^{\infty}\left|G_{1}(r)\right| \int_{r}^{\infty}\left|G_{2}(s)\right| \cdots \int_{u}^{\infty}\left|G_{n-2}(v)\right| \int_{v}^{\infty}\left|G_{n-1}(w)\right| \gamma(w) d w d v d r<\infty
$$

Then there exists a solution $\left(x_{1}, x_{2}, \ldots, x_{n}\right)$ of $(\mathrm{A})$ such that $x_{1}(t) \rightarrow I, x_{i}(t) \rightarrow 0, i=$ $2,3, \ldots, n$, as $t \rightarrow \infty$. Furthermore, there exists $b \geq a$ such that

$$
\begin{gathered}
\left|x_{2}(t)\right| \int_{b}^{t}\left|G_{1}(s)\right| d s \rightarrow 0, \\
\left|x_{3}(t)\right| \int_{b}^{t}\left|G_{2}(s)\right| \int_{b}^{s}\left|G_{1}(u)\right| d u d s \rightarrow 0, \\
\ldots x_{n}(t)\left|\int_{b}^{t}\right| G_{n-1}(r)\left|\int_{b}^{r}\right| G_{n-2}(s)\left|\cdots \int_{b}^{u}\right| G_{1}(v) \mid d v \cdots d r d s \rightarrow 0
\end{gathered}
$$

as $t \rightarrow \infty$. 
To establish Theorem A, consider the integral equation

$$
\begin{aligned}
z(t)= & -\int_{t}^{\infty} G(s) d s \\
& +(-1)^{n} \int_{t}^{\infty} G(r) \int_{r}^{\infty} G_{1}(s) \cdots \int_{u}^{\infty} G_{n-2}(v) \int_{v}^{\infty} G_{n-1}(w) z(w) d w d v \cdots d s d r
\end{aligned}
$$

which is the analog of (9). The proof proceeds as in Theorem 1.

\section{REFERENCES}

1. Philip Hartman, Ordinary differential equations, Wiley, New York, 1964.

2. Philip Hartman and Aurel Wintner, On nonoscillatory linear differential equations, Amer. J. Math. 75 (1953), 717-730.

3. Einar Hille, Nonoscillation theorems, Trans. Amer. Math. Soc. 64 (1948), 234-252.

4. Carl H. Rasmussen, Oscillation and asymptotic behavior of systems of ordinary linear differential equations, Trans. Amer. Math. Soc. 256 (1979), 1-48.

5. Warren E. Shreve, Asymptotic behavior in a second order linear matrix differential equation, J. Differential Equations 9 (1971), 13-24.

6. Aurel Wintner, Asymptotic integrations of the adiabatic oscillator in its hyperbolic range, Duke Math. J. 15 (1948), 55-67.

7. __ On almost free linear motions, Amer. J. Math. 71 (1949), 595-602.

Department of Mathematics, University of Connecticut, Storrs, Connecticut 06268 